\title{
A common data model to assess cardiovascular hospitalization and mortality in atrial fibrillation patients using administrative claims and medical records
}

\author{
This article was published in the following Dove Press journal: \\ Clinical Epidemiology \\ 12 January 2015 \\ Number of times this article has been viewed
}

\author{
Mary P Panaccio' \\ Gordon Cummins ${ }^{2}$ \\ Charles Wentworth ${ }^{3}$ \\ Stephan Lanes ${ }^{4}$ \\ Shannon L Reynolds ${ }^{5}$ \\ Matthew W Reynolds ${ }^{3}$ \\ Raymond Miao' \\ Andrew Koren' \\ 'US Medical Affairs, Sanofi, \\ Bridgewater, NJ, USA; ${ }^{2}$ Health \\ Engagement and Communications, \\ Quintiles, Durham, NC, USA; ${ }^{3}$ Evidera, \\ Lexington, MA, USA; ${ }^{4}$ HealthCore Inc., \\ Andover, MA, USA; ${ }^{5}$ Comprehensive \\ Health Insights, Louisville, KY, USA
}

Correspondence: Mary P Panaccio Evidence Based Medicine, US Medical Affairs, Sanofi, 55 Corporate Drive, Bridgewater, NJ 08807, USA

Tel + I 9089814396

Fax + I 9082037765

Email mary.panaccio@sanofi.com
Purpose: Atrial fibrillation/flutter (AF) is frequently associated with cardiovascular comorbidities. Observational health care databases are commonly used for research purposes in studies of quality of care, health economics, outcomes research, drug safety, and epidemiology. This retrospective cohort study applied a common data model to administrative claims data (Truven Health Analytics MarketScan ${ }^{\circledR}$ claims databases [MS-Claims]) and electronic medical records data (Geisinger Health System's MedMining electronic medical record database [MG-EMR]) to examine the risk of cardiovascular hospitalization and all-cause mortality in relation to clinical risk factors in recent-onset $\mathrm{AF}$ and to assess the consistency of analyses for each data source.

Methods: Cohorts of patients with newly diagnosed AF ( $n=105,262$ [MS-Claims] and n=3,919 [MG-EMR]) and demographically similar patients without AF ( $\mathrm{n}=105,262$ [MS-Claims] and $\mathrm{n}=3,872$ [MG-EMR]) were followed from the qualifying AF diagnosis until cardiovascular hospitalization, death, database disenrollment, or study completion. A common data model standardized the data in structure, format, content, and nomenclature to allow for systematic assessment and comparison of outcomes from two disparate data sets.

Results: In both databases, AF patients had greater overall baseline comorbidity and higher incidence rates of cardiovascular hospitalization (threefold higher) and all-cause mortality ( $46 \%$ higher) than non-AF patients. For AF patients, incidence rates of cardiovascular hospitalization and all-cause mortality were increased by the concomitant presence of coronary disease, chronic obstructive pulmonary disease, and stroke at baseline. Overall, the pattern of cardiovascular hospitalization in the MS-Claims database was similar to that in the MG-EMR database. Compared with the MS-Claims database, the use of cardiovascular medications and the capture of certain comorbidities among AF patients appeared to be higher in the MG-EMR data set.

Conclusion: Similar standardized analyses across EMR and Claims databases were consistent in the association of AF with acute morbidity and an increased risk of all-cause mortality. Areas of inconsistency were due to differences in underlying population demographics and cardiovascular risks and completeness of certain data fields.

Keywords: atrial fibrillation, cardiovascular hospitalization, common data models, epidemiology, observational databases

\section{Introduction}

Atrial fibrillation affects an estimated 3.0 million adults in the United States ${ }^{1}$ and is the most common form of cardiac arrhythmia encountered in clinical practice. Atrial flutter is less prevalent than atrial fibrillation but is a frequent precursor of atrial fibrillation, and the two conditions are often considered in conjunction. ${ }^{2}$ Non-valvular 
atrial fibrillation/flutter (AF) increases the risk of ischemic stroke fivefold, ${ }^{3-5}$ while chronic tachycardia associated with AF can promote left ventricular dysfunction and heart failure. ${ }^{6}$ Further, AF doubles the risk of cardiovascular and all-cause mortality, independently of anticoagulation status and underlying comorbidity. ${ }^{6-8}$ Yearly in the United States, there are an estimated 479,000 hospitalizations with AF as the primary diagnosis and more than 100,000 AF-related deaths. ${ }^{9}$ Most hospitalizations in patients with AF are due to rapid heart rate, heart failure, and stroke. ${ }^{10,11}$ Over the past 30 years, there has been a steady rise in the annual number of AF-related hospital admissions and associated deaths in the United States, ${ }^{12-14}$ which may in part be attributed to increases in life expectancy and changes in health care delivery. ${ }^{13-16}$ Nationally, AF-related health care costs are estimated to range from $\$ 6.0$ billion (direct costs) to $\$ 26.0$ billion (direct and indirect costs) annually, ${ }^{17}$ with hospitalization being the main cost driver. ${ }^{18,19}$

Observational health care databases provide an opportunity to examine the consistency of associations between $\mathrm{AF}$ and cardiovascular hospitalization morbidity measures in real-world clinical practice settings.

The purpose of this study was to 1) standardize methodologies and coding algorithms via a common data model (CDM) across two disparate types of data sources, a) administrative claims data and b) electronic medical records data (EMR), and 2) understand how underlying differences in baseline characteristics across populations affect the observed risk of cardiovascular hospitalization and all-cause mortality in relation to clinical risk factors in recent-onset AF.

\section{Methods}

\section{Data sources}

The study utilized two US health care system databases: 1) the Truven Health Analytics MarketScan ${ }^{\circledR}$ claims databases (MS-Claims) and 2) the Geisinger Health System's MedMining EMR database (MG-EMR). Both data sources are widely used for epidemiologic and health economics and outcomes research. ${ }^{20-25}$ Patient data were de-identified in compliance with the Health Insurance Portability and Accountability Act of 1996.

The MS-Claims databases capture individual-level clinical utilization, expenditures, and enrollment across inpatient, outpatient, prescription drug, and carve-out services from $\sim 45$ large employers, health plans, and government and public organizations for $\sim 64$ million individuals. The databases include commercially insured employees and their dependents, early retirees, COBRA (Consolidated Omnibus
Budget Reconciliation Act) - and Medicare-eligible retirees with employer-provided Medicare Supplemental plans, and eleven state Medicaid plans.

The MG-EMR database provides information on patient demographics, inpatient and outpatient care, pharmacy prescriptions, laboratory results, procedures, diagnoses, lifestyle measures, and vital status of $\sim 3.8$ million individuals (1996 to present) covered by the Geisinger Health System. The Geisinger Health System provides care to patients of all ages (including Medicare-eligible patients) in Central Pennsylvania, USA. Vital status and date of death were ascertained via linkage with the Social Security Administration's Death Master files. These files report vital status but not cause of death.

\section{Patient selection and study design}

This cohort study included patients with newly diagnosed AF and a comparator group of demographically similar patients without AF. To minimize variability between the databases and enable systematic analyses across disparate databases, a CDM was used to standardize these data in structure, format, content, and nomenclature, as previously published. ${ }^{26}$

For inclusion in this study, patients were required to have $\geq 1$ inpatient AF diagnosis (International Classification of Diseases, Ninth Revision [ICD-9] diagnostic code 427.31 or 427.32) or two outpatient AF diagnoses on separate days within 30 consecutive days, after an interval of $\geq 364$ days with no AF diagnosis, between January 2003 and December 2009 (MS-Claims) or January 2005 to September 2010 (MG-EMR) (Figure 1). Enrolled patients without an AF diagnosis who had $\geq 1$ non-AF-related inpatient encounter or $\geq 2$ non-AF-related outpatient encounters over the corresponding period were matched to AF patients by sex, birth date ( \pm 5 years), geographic region (MS-Claims only - all EMR patients were from the same region), and insurance type to form a control group. Patients were required to have $\geq 364$ days of continuous database enrollment before the qualifying index date ( $\mathrm{AF}$ diagnosis date for $\mathrm{AF}$ patients and a qualifying encounter date for non-AF comparators), which was considered the 1-year baseline period. Non-AF patients did not have any diagnosis of AF during the baseline period or at the qualifying encounter date. The day after the index date indicated the beginning of follow-up, and the patients required $\geq 1$ day of follow-up to be included (Figure 2). Patients were excluded if they were $<40$ years old on their index date, had a diagnosis of hyperthyroidism or valvular heart disease $<90$ days before their index date, or 


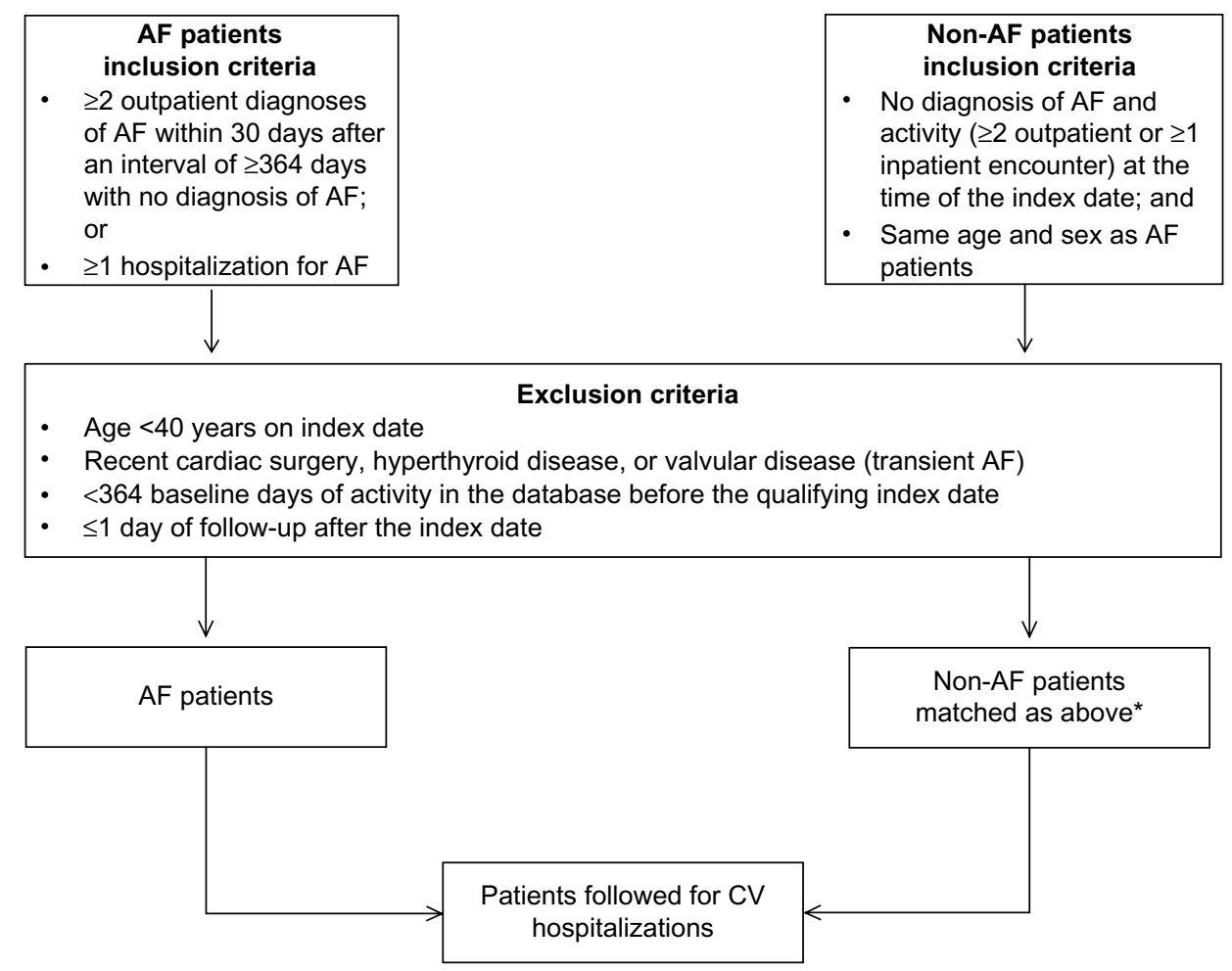

Figure I Selection of AF and non-AF patients (MS-Claims and MG-EMR data sets).

Note: *Non-AF comparators matched on sex, age ( \pm 5 years), region (Northeast, North central, West, and South), health plan (commercial, Medicare, and Medicaid). Abbreviations: AF, atrial fibrillation/flutter; CV, cardiovascular; MG-EMR, Geisinger Health System's MedMining electronic medical record database; MS-Claims, Truven Health Analytics MarketScan ${ }^{\circledR}$ claims databases.

had undergone cardiac surgery $<364$ days before their index date (Figure 1). Patients with chronic AF were further identified by excluding patients with transient AF, defined as an $\mathrm{AF}$ diagnosis with evidence of hyperthyroidism (signified by an ICD-9 diagnosis code for hyperthyroidism or an outpatient prescription claim for methimazole or propylthiouracil in

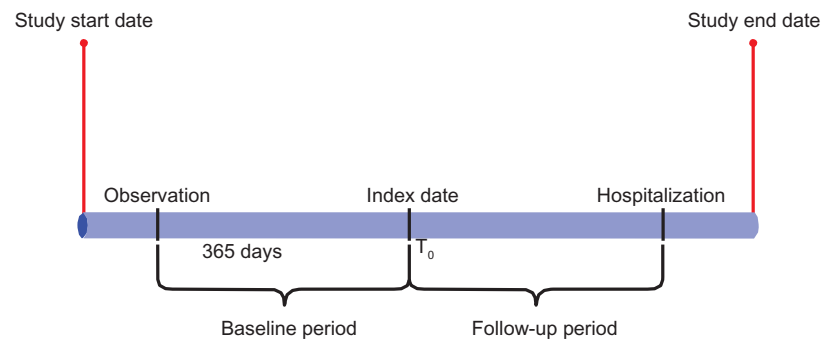

Figure 2 Study design and chronology.

Notes: Index date $\left(T_{0}\right)$ : date of the first qualifying AF diagnosis. For non-AF patients, index date was the time of the qualifying encounter. Baseline period: 365 baseline days of continuous enrollment in the database before the qualifying index date and inclusive of qualifying index diagnosis date. Follow-up period: time during which the patient contributed person-time to the denominator of the incidence rate of cardiovascular hospitalization and was eligible to be included as a case in the numerator of the incidence rate. Start of follow-up is the day after index date $\left(T_{0}\right)$ End of follow-up is the earliest of the following dates: 1 ) end of the study calendar period (3I December 2009 or 30 September 2010); 2) occurrence of a study end point (cardiovascular hospitalization admission date, AF hospitalization admission date); 3) exit from the database (eg, end date in the database).

Abbreviation: AF, atrial fibrillation/flutter. the 12-month period before the index diagnosis), or an $\mathrm{AF}$ diagnosis after recent cardiac surgery (Figure $3 \mathrm{~A}$ and $\mathrm{B}$ ).

\section{Baseline characteristics and outcome measures}

Data on patient demographics, clinical, and treatment characteristics (Charlson Comorbidity Index ${ }^{27}$ ) were ascertained over the 12-month preindex period. For variables with multiple database entries, values closest to the index date were used. Comorbidities of interest were identified from relevant ICD-9 diagnostic codes; cardiovascular medications were identified from National Drug Codes (MS-Claims) or therapeutic codes and string searches (MG-EMR).

Study end points included cardiovascular hospitalization, all-cause mortality (MG-EMR data set only), and newly recorded morbidity events/conditions (such as myocardial infarction [MI] or cerebrovascular disease) during the postindex follow-up. Patient follow-up extended from the index date until occurrence of cardiovascular hospitalization or death, termination of database enrollment, or completion of the study period, whichever occurred first. Cardiovascular hospitalizations were categorized by cause, as determined from the primary discharge diagnosis (Table 1). 


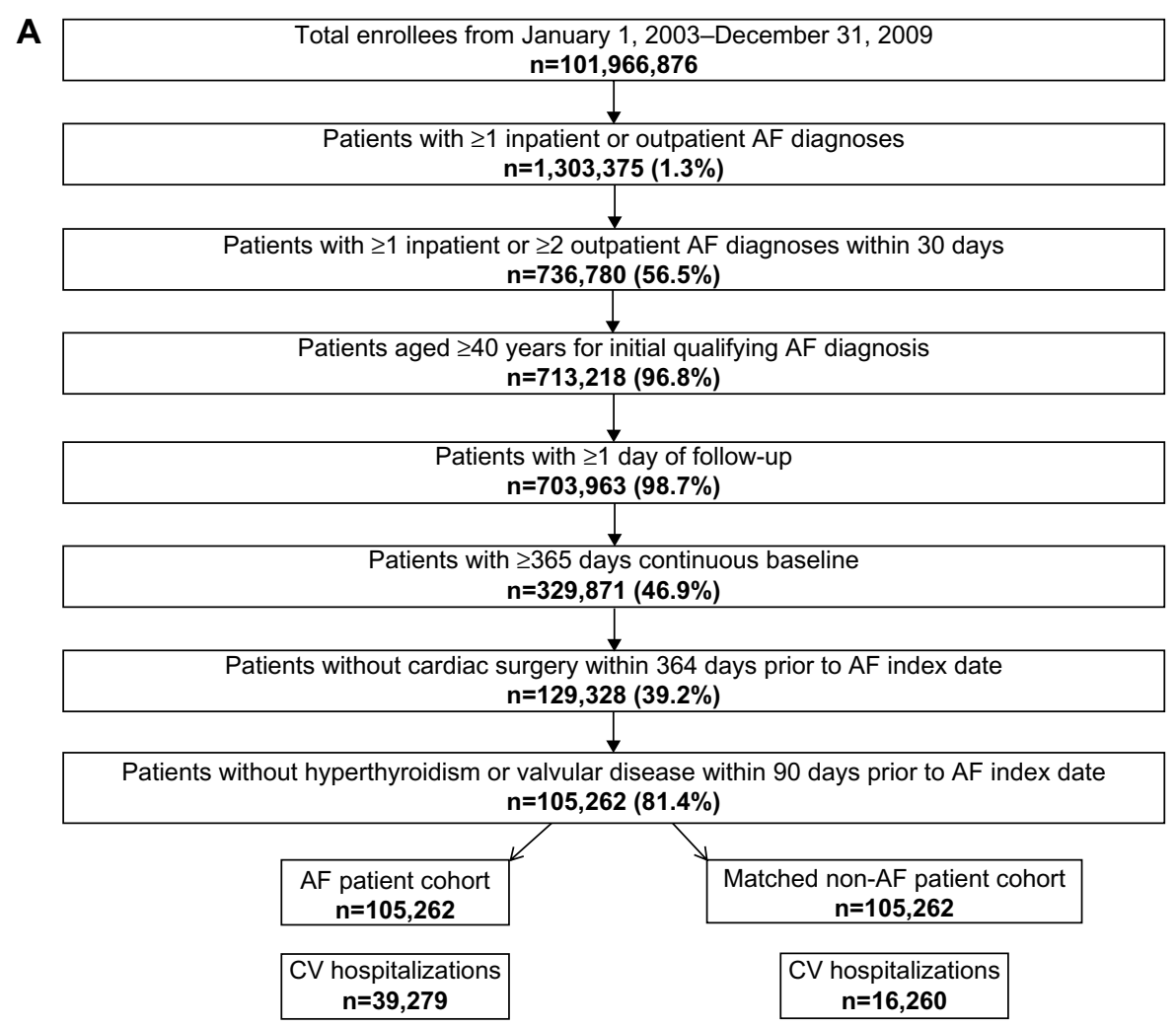

B

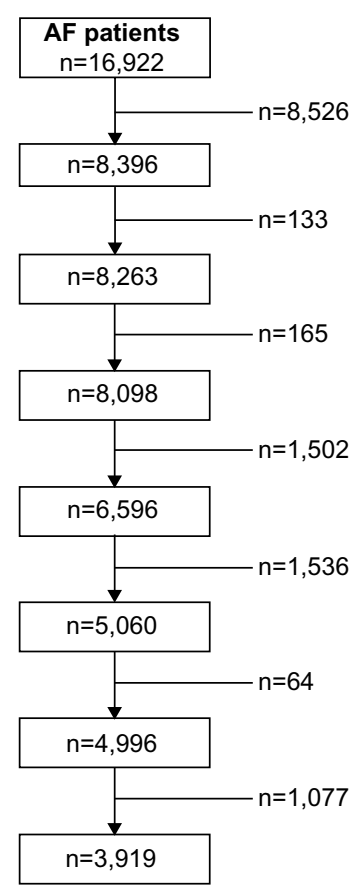

Patients for potential

inclusion in analytic cohort

matched from January 2005-

September 2010 on:

- Age \pm 5 years

- Sex

1 inpatient or 2 outpatient diagnoses within 30 days

Age $>40$ years at index

$\geq 1$ day of follow-up

>365 days of baseline enrollment

$n=3,238$

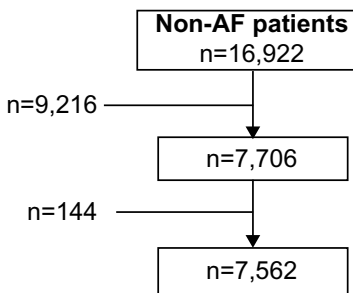

$n=315$

No cardiac surgery within 364 days prior to AF index date

No hyperthyroidism within 90 days prior to index date

No valvular disease within 90 days prior to index date

Figure 3 Patient flow.

Notes: (A) MS-Claims data set. (B) MG-EMR data set.

Abbreviations: AF, atrial fibrillation/flutter; Cl, confidence interval; CV, cardiovascular; MG-EMR, Geisinger Health System's MedMining electronic medical record database; MS-Claims, Truven Health Analytics MarketScan ${ }^{\circledast}$ claims databases. 
Table I Causes of cardiovascular hospitalization and the corresponding ICD-9 diagnostic codes

\begin{tabular}{|c|c|}
\hline Cause of cardiovascular hospitalization & ICD-9 code \\
\hline All-cause cardiovascular disease & $390 . x-459 . x$ \\
\hline Atrial fibrillation/flutter & $427.31,427.32$ \\
\hline Rheumatic heart disease & $\begin{array}{l}391 . x \\
393 . x-398 . x\end{array}$ \\
\hline Hypertensive disease & $\begin{array}{l}401 . x-405 . x \\
437.2\end{array}$ \\
\hline $\begin{array}{l}\text { Coronary heart disease (acute myocardial infarction, } \\
\text { acute/sub-acute coronary syndrome, and angina } \\
\text { pectoris) }\end{array}$ & $4|0 . x-4| 4 \cdot x$ \\
\hline Pulmonary circulatory disease & $4|5 . x-4| 7 . x$ \\
\hline $\begin{array}{l}\text { Other forms of heart disease (acute pericarditis, } \\
\text { acute/sub-acute endocarditis, acute myocarditis, } \\
\text { other diseases of pericardium, other diseases of } \\
\text { endocardium, cardiomyopathy, conduction disorders, } \\
\text { cardiac dysrhythmias, and ventricular arrhythmia or } \\
\text { cardiac arrest) }\end{array}$ & $420 . x-429 . x$ \\
\hline Heart failure & $398.91,428 . x$ \\
\hline Cerebrovascular disease & $430 . x-438 . x$ \\
\hline $\begin{array}{l}\text { Diseases of the arteries, arterioles, and capillaries } \\
\text { (including atherosclerosis) }\end{array}$ & $440 . x-448 . x$ \\
\hline $\begin{array}{l}\text { Diseases of the veins and lymphatics, and other } \\
\text { disease of the circulatory system (including phlebitis, } \\
\text { thrombophlebitis, and portal vein thrombosis) }\end{array}$ & $451 . x-459 . x$ \\
\hline
\end{tabular}

Abbreviation: ICD-9, International Classification of Diseases, Ninth Revision.

\section{Statistical analysis}

Data were summarized using descriptive statistics. Intercohort comparisons of outcomes were summarized using prevalence odds ratios (baseline comorbidity prevalence) or incidence rate ratios (IRRs) (comorbidity, cardiovascular hospitalization, and all-cause mortality incidence rates during follow-up) with 95\% confidence intervals (CIs).

Baseline prevalence rates of comorbidity and medication use were determined for each cohort and prevalence odds ratios (AF cohort:non-AF cohort) and 95\% CIs calculated. Incidence rates of newly recorded morbidity events/ conditions, cardiovascular hospitalization, and all-cause mortality during follow-up were calculated for each patient cohort, and IRRs and 95\% CIs were derived. Additional analyses performed only for the MS-Claims data set included comparisons of incidence rates of cardiovascular hospitalization among AF patients stratified by 1) presence/absence of selected baseline comorbidities; 2) baseline $\mathrm{CHADS}_{2}$ (congestive heart failure, hypertension, age $\geq 75$ years, diabetes mellitus, and prior stroke/transient ischemic attack [TIA]) score ${ }^{28}$ and 3) baseline $\mathrm{CHA}_{2} \mathrm{DS}_{2}$-VASc (congestive heart failure, hypertension, age $\geq 75$ years, diabetes mellitus, prior stroke/TIA, vascular disease, age 65-74 years, sex category) score. ${ }^{29}$ Kaplan-Meier curves of cumulative incidence of cardiovascular hospitalization over time were derived using the life-table approach. Analysis procedures to create the cohorts and develop the analytic variables were performed using SAS 9.1. Analyses were performed in STATA version 7 using the STIR procedure to compute point estimates and 95\% CIs for rate ratios and the STS GRAPH procedure to generate Kaplan-Meier survival functions.

\section{Results}

\section{Demographic and baseline clinical characteristics}

A total of 1,303,375 individuals were identified in the MSClaims databases (2003-2009) as having $\geq 1$ inpatient or $\geq 2$ outpatient diagnoses of AF, of whom 105,262 patients satisfied the study eligibility criteria and were matched (by sex, birth date [ \pm 5 years], and insurance type) with 105,262 non-AF patients (Figure $3 \mathrm{~A}$ ). The AF and non-AF cohorts were therefore of similar age at index date (mean 74 years), sex (49\% male), geographic distribution, and insurance type (predominantly Medicare) (Table 2). The duration of postindex follow-up for the AF and non-AF cohorts was similar (mean [standard deviation] 1.7 [1.6] and 1.9 [1.5] years, respectively).

Of 8,396 patients with $\geq 1$ inpatient or $\geq 2$ outpatient diagnoses of AF identified in the MG-EMR database (2005-2010), 3,919 patients were eligible for study inclusion and were matched with 3,872 non-AF patients by sex and birth date ( \pm 5 years) (Figure $3 \mathrm{~B})$. The AF and non-AF cohorts were of similar age (mean 73 and 71 years, respectively), sex (53\% and $57 \%$ male, respectively), and ethnicity (predominantly Caucasian) (Table 2). The duration of postindex follow-up was similar for AF and non-AF cohorts (mean [standard deviation] 1.9 [1.4] and 2.0 [1.4] years, respectively). In the MG-EMR data set, obesity (body mass index [BMI] $>30 \mathrm{mg}$ / $\mathrm{kg}^{2}$ ) was more prevalent in AF than in non-AF patients $(47.2 \%$ vs $36.6 \%$ ) (Table 2). Obesity (identified by ICD-9 code) was less prevalent in both patient groups (Table 3) (suggesting possible underreporting), but showed similar patterns across databases, being more frequent in $\mathrm{AF}$ than in non-AF patients (MS-Claims: $2.9 \%$ vs $0.6 \%$; MG-EMR: $11.5 \%$ vs $1.5 \%$ ).

Overall, AF patients had greater comorbidity burden at baseline (Table 2). Use of cardiovascular medications was more prevalent among AF than non-AF patients.

Baseline comorbidity burden among AF patients was similar for many major cardiovascular events/conditions (MI, heart failure, stroke, cardiac hypertrophy, structural heart disease, and major bleeding), chronic obstructive pulmonary disease (COPD), and hyperthyroidism (Table 3 ). 
Table 2 Patient population: baseline characteristics by database

\begin{tabular}{|c|c|c|c|c|}
\hline \multirow[t]{2}{*}{ Characteristics } & \multicolumn{2}{|l|}{ MS-Claims } & \multicolumn{2}{|l|}{ MG-EMR } \\
\hline & AF cohort & Non-AF cohort & AF cohort & Non-AF cohort \\
\hline Number of patients & 105,262 & 105,262 & 3,919 & 3,872 \\
\hline Male (\%) & 49.2 & 49.2 & 52.8 & 56.8 \\
\hline \multicolumn{5}{|l|}{ Age (years) } \\
\hline Mean (range) & $74.4(40-107)$ & $73.6(40-107)$ & 72.7 (40-89) & 71.1 (40-89) \\
\hline \multicolumn{5}{|l|}{ Age category (\%) } \\
\hline $40-49$ years & 4.3 & 4.3 & 3.9 & 4.4 \\
\hline 50-59 years & 10.0 & 10.0 & 11.0 & 10.7 \\
\hline $60-69$ years & 16.9 & 16.9 & 20.2 & 20.6 \\
\hline 70-79 years & 28.4 & 28.4 & 31.9 & 40.2 \\
\hline$\geq 80$ years & 40.3 & 40.3 & 32.7 & 24.2 \\
\hline \multicolumn{5}{|l|}{ Race (\%) } \\
\hline Caucasian & NA & NA & 99.1 & 97.9 \\
\hline African-American & & & 0.5 & 0.4 \\
\hline Hispanic & & & 0.1 & 0.5 \\
\hline Other & & & 0.2 & 0.9 \\
\hline Unknown & & & 0.1 & 0.3 \\
\hline \multicolumn{5}{|l|}{ Insurance type (\%) } \\
\hline Commercial & 20.7 & 20.7 & 71.3 & 62.7 \\
\hline Medicare & 67.7 & 67.7 & 26.4 & 32.7 \\
\hline Medicaid & 11.6 & 11.6 & 0.7 & 1.0 \\
\hline \multicolumn{5}{|l|}{ Geographic location (\%) } \\
\hline Northeast & 9.1 & 9.1 & 100 & 100 \\
\hline North central & 33.7 & 33.7 & & \\
\hline South & 24.7 & 24.7 & & \\
\hline West & 20.6 & 20.6 & & \\
\hline Unknown & 0.2 & 0.2 & & \\
\hline \multicolumn{5}{|l|}{ Index AF diagnosis (\%) } \\
\hline Inpatient & 56.7 & 56.7 & 49.5 & 49.5 \\
\hline Outpatient & 43.3 & 43.3 & 50.5 & 50.5 \\
\hline \multicolumn{5}{|c|}{ Charlson Comorbidity Index (\%) } \\
\hline 0 & 31.4 & 64.5 & 23.1 & 59.1 \\
\hline 1 & 25.0 & 18.6 & 17.5 & 12.5 \\
\hline 2 & 16.1 & 8.5 & 16.5 & 17.7 \\
\hline 3 & 11.0 & 4.4 & 13.2 & 4.9 \\
\hline$\geq 4$ & 16.5 & 4.1 & 29.5 & 5.8 \\
\hline \multicolumn{5}{|l|}{ BMI $(\%)^{\mathrm{a}}$} \\
\hline$<18.5 \mathrm{~kg} / \mathrm{m}^{2}$ & NA & NA & 1.2 & 0.7 \\
\hline $18.5-24.9 \mathrm{~kg} / \mathrm{m}^{2}$ & & & 15.0 & 9.5 \\
\hline $25.0-30.0 \mathrm{~kg} / \mathrm{m}^{2}$ & & & 24.6 & 14.3 \\
\hline$>30.0 \mathrm{~kg} / \mathrm{m}^{2}$ & & & 36.4 & 14.2 \\
\hline Missing & & & 22.7 & 61.3 \\
\hline Mean (SD) & & & $31.0(8.0)$ & $28.9(6.5)$ \\
\hline Median (range) & & & $29.6(1.5-76.0)$ & $28.2(2.3-71.8)$ \\
\hline \multicolumn{5}{|l|}{ Medications (\%) } \\
\hline$\beta$-blockers & 44.1 & 21.5 & 68.3 & 23.4 \\
\hline $\mathrm{CCBs}$ & 31.2 & 17.6 & 35.3 & 9.8 \\
\hline Digoxin & 15.4 & 1.6 & 18.9 & 1.5 \\
\hline ACE inhibitors/ARBs & 13.8 & 9.5 & 41.0 & 20.8 \\
\hline Statins & 33.9 & 29.9 & $4 I .1$ & 24.6 \\
\hline Anticoagulants & 28.9 & 2.3 & 59.7 & 5.9 \\
\hline Aspirin & 2.3 & 1.4 & 38.0 & 25.6 \\
\hline
\end{tabular}

Note: aBMI data were only available for the MG-EMR data set (3,028 AF patients and I,500 non-AF patients).

Abbreviations: $\mathrm{ACE}$, angiotensin converting enzyme; $\mathrm{AF}$, atrial fibrillation/flutter; $\mathrm{ARB}$, angiotensin receptor blocker; $\mathrm{BMI}$, body mass index; CCB, calcium channel blocker; MG-EMR, Geisinger Health System's MedMining electronic medical record database; MS-Claims, Truven Health Analytics MarketScan ${ }^{\circledR}$ claims databases; NA, not available; $\mathrm{SD}$, standard deviation. 
Table 3 Prevalence of comorbidity during the baseline period among AF and non-AF patient cohorts by database

\begin{tabular}{|c|c|c|c|c|c|c|}
\hline \multirow{2}{*}{$\begin{array}{l}\text { Prevalence of } \\
\text { comorbidity during } \\
\text { baseline period }\end{array}$} & \multicolumn{3}{|l|}{ MS-Claims } & \multicolumn{3}{|l|}{ MG-EMR } \\
\hline & $\begin{array}{l}\text { AF cohort } \\
(n=105,262), \\
N(\%)\end{array}$ & $\begin{array}{l}\text { Non-AF cohort } \\
(n=105,262), \\
N(\%)\end{array}$ & $\begin{array}{l}\text { Prevalence } \\
\text { odds ratio } \\
(95 \% \mathrm{CI})\end{array}$ & $\begin{array}{l}\text { AF cohort } \\
(n=3,9 \mid 9), \\
N(\%)\end{array}$ & $\begin{array}{l}\text { Non-AF cohort } \\
(n=3,872) \\
N(\%)\end{array}$ & $\begin{array}{l}\text { Prevalence } \\
\text { odds ratio } \\
(95 \% \mathrm{Cl})\end{array}$ \\
\hline Myocardial infarction & $3,825(3.6 \%)$ & $302(0.3 \%)$ & I3.I (II.7-|4.7) & $98(2.5 \%)$ & $3(0.1 \%)$ & $33.1(11.0-163.2)$ \\
\hline Heart failure & $25,283(24.0 \%)$ & $3,49 \mid(3.3 \%)$ & $9.2(8.9-9.6)$ & $927(23.7 \%)$ & $56(1.4 \%)$ & $21.1(16.0-28.3)$ \\
\hline Pulmonary embolism & $\mathrm{I}, 827(\mathrm{I} .7 \%)$ & $225(0.2 \%)$ & $8.2(7.2-9.5)$ & 125 (3.2\%) & II (0.3\%) & $11.6(6.2-23.8)$ \\
\hline Cardiac hypertrophy & $9,705(9.2 \%)$ & $\mathrm{I}, 44 \mathrm{I}(\mathrm{I} .4 \%)$ & $7.3(6.9-7.7)$ & 358 (9.1\%) & $16(0.4 \%)$ & $24.2(14.7-42.9)$ \\
\hline Obesity $^{\mathrm{a}}$ & $3,066(2.9 \%)$ & $655(0.6 \%)$ & $4.8(4.4-5.2)$ & 450 (II.5\%) & $60(1.5 \%)$ & $8.2(6.3-10.8)$ \\
\hline Obesity $^{b}$ & NA & NA & NA & $\mathrm{I}, 428(47.2 \%)$ & $549(36.6 \%)$ & $3.5(3.1-3.9)$ \\
\hline Syncope & $8,745(8.3 \%)$ & $2,305(2.2 \%)$ & $4.1(3.9-4.2)$ & 249 (6.4\%) & $43(1.1 \%)$ & $6.0(4.3-8.6)$ \\
\hline Structural heart disease & 33,417 (31.7\%) & $8,320(7.9 \%)$ & $5.4(5.3-5.6)$ & $\mathrm{I}, 223(3 \mathrm{I} .2 \%)$ & $95(2.5 \%)$ & $18.0(14.5-22.3)$ \\
\hline Coronary heart disease & 30,471 (28.9\%) & $11,422(10.9 \%)$ & $3.4(3.3-3.4)$ & $1,279(32.6 \%)$ & $225(5.8 \%)$ & $7.9(6.7-9.2)$ \\
\hline COPD & $19,43 \mid(\mid 8.5 \%)$ & $6,688(6.4 \%)$ & $3.3(3.2-3.4)$ & $68 I(17.4 \%)$ & $107(2.8 \%)$ & $7.4(6.0-9.2)$ \\
\hline Stroke & 19,748 (18.8\%) & $7,063(6.7 \%)$ & $3.2(3.1-3.3)$ & $814(20.8 \%)$ & $203(5.2 \%)$ & $4.7(4.0-5.6)$ \\
\hline Sleep apnea & $4,455(4.2 \%)$ & I,795 (I.7\%) & $2.6(2.4-2.7)$ & $280(7.1 \%)$ & $25(0.6 \%)$ & II.8 (7.8-18.7) \\
\hline Hypertension & $56,602(53.8 \%)$ & $35,079(33.3 \%)$ & $2.3(2.3-2.4)$ & $2,720(69.4 \%)$ & 645 (I6.7\%) & II.4 (I0.2-12.7) \\
\hline Major bleeding & 33,360 (31.7\%) & $18,578(17.6 \%)$ & $2.2(2.1-2.2)$ & I,09। (27.8\%) & $423(10.9 \%)$ & $3.2(2.8-3.6)$ \\
\hline Diabetes & 22,642 (21.5\%) & $13,698(13.0 \%)$ & $1.8(1.8-1.9)$ & I, I I 4 (28.4\%) & $302(7.8 \%)$ & $4.7(4.1-5.4)$ \\
\hline Valvular heart disease & $5,192(4.9 \%)$ & $2,983(2.8 \%)$ & $1.8(1.7-1.9)$ & 110 (2.8\%) & $7(0.2 \%)$ & I5.5 (7.3-39.5) \\
\hline Hyperthyroidism & $372(0.4 \%)$ & $312(0.3 \%)$ & I.2(I.0-I.4) & $16(0.4 \%)$ & $9(0.2 \%)$ & $1.8(0.7-4.5)$ \\
\hline
\end{tabular}

Notes: a Obesity defined by ICD-9 codes 278.00-278.02; 'obesity defined by BMI $>30 \mathrm{~kg} / \mathrm{m}^{2}$; based on BMI data available for the MG-EMR database (3,028 patients [77.3\%] in the AF cohort and I,500 patients [38.7\%] in the non-AF cohort).

Abbreviations: AF, atrial fibrillation/flutter; BMI, body mass index; $\mathrm{Cl}$, confidence interval; COPD, chronic obstructive pulmonary disease; ICD, International Classification of Diseases, Ninth Revision; MG-EMR, Geisinger Health System’s MedMining electronic medical record database; MS-Claims, Truven Health Analytics MarketScan ${ }^{\circledR}$ claims databases; NA, not available.

Hypertension was the most prevalent of the baseline comorbidities investigated among AF patients (Table 3 ). In contrast, heart failure, structural heart disease, COPD, hypertension, valvular heart disease, and diabetes were reported less frequently among non-AF patients (Table 3 ). The leading comorbidities showing the most pronounced differences in prevalence between $\mathrm{AF}$ and non-AF patients across databases included MI, heart failure, cardiac hypertrophy, pulmonary embolism, and structural heart disease (Table 3).

\section{Comparison between databases for common analyses}

Patients in the MS-Claims data set had a greater overall mean age of 74 years (range 40-107) versus patients in the MGEMR data set (mean age 71.9 years; range 40-89), due to a higher proportion of patients in the $\geq 80$ years age category (40.3\% vs $28.5 \%$ ) (Table 2 ).

Similarities across the two databases were observed for postindex risk of cardiovascular hospitalization and new morbidities as well as specific baseline comorbidities. The incidence rate of all-cause cardiovascular hospitalization was 3.4-fold higher in AF than in non-AF patients (Table 4). Admissions for cardiac dysrhythmias (other than AF), cardiomyopathy, other forms of heart disease, heart failure, and rheumatic heart disease were $\geq 4$ times more frequent in AF than non-AF patients (Table 4). Admissions for hypertensive disease, coronary heart disease, cerebrovascular disease, conduction disorders, arterial disease, and venous/lymphatic disease were $\sim 2$ times more frequent among $\mathrm{AF}$ patients than non-AF patients (Table 4).

Hospitalization rates among AF patients with and without baseline comorbidity indicated that the presence of baseline heart failure, COPD, coronary heart disease, and MI increased the risk of all-cause cardiovascular hospitalization by $>50 \%$ during the follow-up period (Figure $4 \mathrm{~A}$ and $\mathrm{B})$.

The most frequent newly recorded morbidity events/ conditions among AF patients were hypertension, major bleeding, coronary heart disease, heart failure, valvular heart disease, and stroke (Table 5). The incidence rates of all selected cardiovascular, cardiometabolic, and respiratory comorbidities (apart from diabetes in the MG-EMR database) were significantly higher among AF patients than non-AF patients (Table 5).

Differences across databases were observed in certain baseline medications and co-morbidities, and in the incidence of new cardiovascular events and hospitalizations. At baseline, patients from the MG-EMR database had higher 
Table 4 Incidence rates of cardiovascular hospitalization during the postindex period among AF and non-AF cohorts by database

\begin{tabular}{|c|c|c|c|c|c|c|}
\hline \multirow{3}{*}{$\begin{array}{l}\text { Hospital discharge diagnosis } \\
\text { (specific cardiovascular cause) }\end{array}$} & \multicolumn{3}{|l|}{ MS-Claims } & \multicolumn{3}{|l|}{ MG-EMR } \\
\hline & \multicolumn{2}{|c|}{$\begin{array}{l}\text { Incidence rate ( } \mathrm{n} \text { per } \\
\mathrm{I}, 000 \text { patient-years) }\end{array}$} & \multirow{2}{*}{$\begin{array}{l}\text { Incidence } \\
\text { rate ratio } \\
(95 \% \mathrm{Cl})\end{array}$} & \multicolumn{2}{|c|}{$\begin{array}{l}\text { Incidence rate (n per } \\
\text { I,000 patient-years) }\end{array}$} & \multirow{2}{*}{$\begin{array}{l}\text { Incidence } \\
\text { rate ratio } \\
(95 \% \mathrm{Cl})\end{array}$} \\
\hline & $\begin{array}{l}\text { AF cohort } \\
(n=105,262)\end{array}$ & $\begin{array}{l}\text { Non-AF cohort } \\
(n=105,262)\end{array}$ & & $\begin{array}{l}\text { AF cohort } \\
(n=3,9 \mid 9)\end{array}$ & $\begin{array}{l}\text { Non-AF cohort } \\
(n=3,872)\end{array}$ & \\
\hline $\begin{array}{l}\text { All cardiovascular disease } \\
\text { (ICD-9: } 390 . x-459 . x)\end{array}$ & 311.3 & 91.4 & $3.4(3.4-3.5)$ & 341.2 & 100.4 & $3.4(3.1-3.7)$ \\
\hline $\begin{array}{l}\text { Atrial fibrillation and flutter } \\
\text { (ICD-9: 427.3x) }\end{array}$ & 175.8 & 0 & - & 169.6 & 0 & - \\
\hline Cardiac dysrhythmias (ICD-9: 427.x) & 201.0 & 13.4 & I5.I (|4.5-15.7) & 207.1 & 22.4 & $9.3(7.9-11.0)$ \\
\hline Cardiomyopathy (ICD-9: 425.x) & 17.6 & 2.8 & $6.3(5.8-6.9)$ & 6.1 & 1.5 & $4.2(2.1-8.9)$ \\
\hline $\begin{array}{l}\text { Other forms of heart disease } \\
(\text { ICD-9: } 420 . x-429 . x)^{a}\end{array}$ & 253.6 & 40.3 & $6.3(6.1-6.5)$ & 279.4 & 49.4 & $5.7(5.0-6.4)$ \\
\hline Heart failure (ICD-9: 398.9I, 428.x) & 90.3 & 17.4 & $5.2(5.0-5.4)$ & 78.1 & 14.1 & $5.5(4.5-6.9)$ \\
\hline $\begin{array}{l}\text { Rheumatic heart disease } \\
\text { (ICD-9: 391.x, 393.x-398.x) }\end{array}$ & 9.7 & 2.1 & $4.6(4.1-5.1)$ & 5.9 & 1.5 & $4.0(2.0-8.6)$ \\
\hline $\begin{array}{l}\text { Diseases of veins and lymphatics, } \\
\text { and other diseases of circulatory } \\
\text { system (ICD-9: } 45 \text { I.x-459.x) }\end{array}$ & 30.7 & 10.7 & $2.9(2.7-3.0)$ & 28.7 & 12.9 & $2.2(1.7-2.9)$ \\
\hline $\begin{array}{l}\text { Coronary heart disease } \\
(I C D-9: 4|0 . x-4| 4 . x)^{c}\end{array}$ & 78.5 & 28.5 & $2.8(2.7-2.8)$ & 52.8 & 26.7 & $2.0(1.7-2.4)$ \\
\hline Conduction disorders (ICD-9: 426.x) & 12.4 & 4.9 & $2.5(2.3-2.7)$ & 13.0 & 6.9 & $1.9(1.3-2.7)$ \\
\hline $\begin{array}{l}\text { Diseases of arteries, arterioles, } \\
\text { and capillaries (ICD-9: } 440 . x-448 . x)^{d}\end{array}$ & 21.8 & 8.7 & $2.5(2.4-2.6)$ & 22.6 & 12.1 & $1.9(1.4-2.5)$ \\
\hline $\begin{array}{l}\text { Cerebrovascular disease (ICD-9: } \\
430 . x-438 . x)\end{array}$ & 47.5 & 20.0 & $2.4(2.3-2.5)$ & 61.6 & 31.0 & $2.0(1.7-2.4)$ \\
\hline $\begin{array}{l}\text { Hypertensive disease (ICD-9: } \\
401 . x-405 . x, 437.2)\end{array}$ & 101.0 & 44.7 & $2.3(2.2-2.3)$ & 20.2 & 10.6 & $1.9(1.4-2.5)$ \\
\hline All-cause mortality & NA & NA & NA & 28.1 & 19.3 & $1.5(1.2-1.8)$ \\
\hline $\begin{array}{l}\text { Cardiovascular hospitalization } \\
\text { or all-cause mortality }\end{array}$ & NA & NA & NA & 353.6 & 110.0 & $3.2(2.9-3.5)$ \\
\hline
\end{tabular}

Notes: ancludes acute pericarditis, acute/subacute endocarditis, acute myocarditis, other diseases of pericardium, other diseases of endocardium, cardiomyopathy, conduction

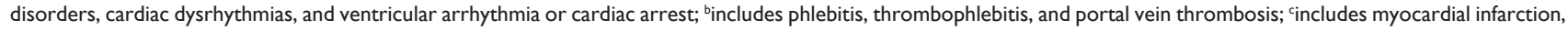

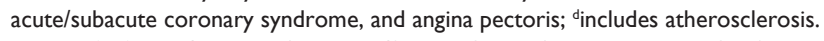

Abbreviations: AF, atrial fibrillation/flutter; Cl, confidence interval; ICD-9, International Classification of Diseases, Ninth Revision; MG-EMR, Geisinger Health System's MedMining electronic medical record database; MS-Claims, Truven Health Analytics MarketScan ${ }^{\circledR}$ claims databases; NA, not available.

cardiovascular medication use than patients in the MSClaims database (Table 2). There were also differences in the capture of comorbidities between AF and non-AF patients, as evidenced by the markedly higher prevalence odds ratios for valvular heart disease, hypertension, and sleep apnea in the MG-EMR data set compared with the MS-Claims data set (Table 3). Among non-AF patients, prevalence rates for most comorbidities were lower in the MG-EMR database than in the MS-Claims database, except for pulmonary embolism and obesity (ICD-9 defined) (Table 3). Among AF patients, the reported prevalence of pulmonary embolism, hypertension, diabetes, obesity, and sleep apnea were higher in MG-EMR data compared with MS-Claims data. The incidence rates of cardiovascular hospitalization from hypertensive disease and cardiomyopathy among AF patients were markedly higher ( $~ 5$ times and $\sim 3$ times, respectively) in the MS-Claims versus MG-EMR data (Table 4). In the MG-EMR data set, the risk of all-cause cardiovascular hospitalization among AF patients was increased by $>50 \%$ by the presence of cardiac hypertrophy, valvular heart disease, pulmonary embolism, or stroke (Figure 4B). Time to onset of all selected new morbidities was shorter for AF than non-AF patients in the MS-Claims data. In contrast, no differences were observed for syncope, MI, pulmonary embolism, and hyperthyroidism in the MG-EMR data.

Times to hospitalization for cardiovascular disease among AF patients and non-AF patients were similar across databases (Figure 5A and B). AF patients experienced more hospitalizations for cardiovascular disease immediately after receiving the initial AF diagnosis, and time-to-event curves for AF and nonAF cohorts continued to diverge over the follow-up period.

\section{Database-specific analysis}

In the MS-Claims analysis, all-cause cardiovascular hospitalization rates for $\mathrm{AF}$ and non-AF patients progressively increased with ascending $\mathrm{CHADS}_{2}$ and $\mathrm{CHA}_{2} \mathrm{DS}_{2}-\mathrm{VASc}$ 

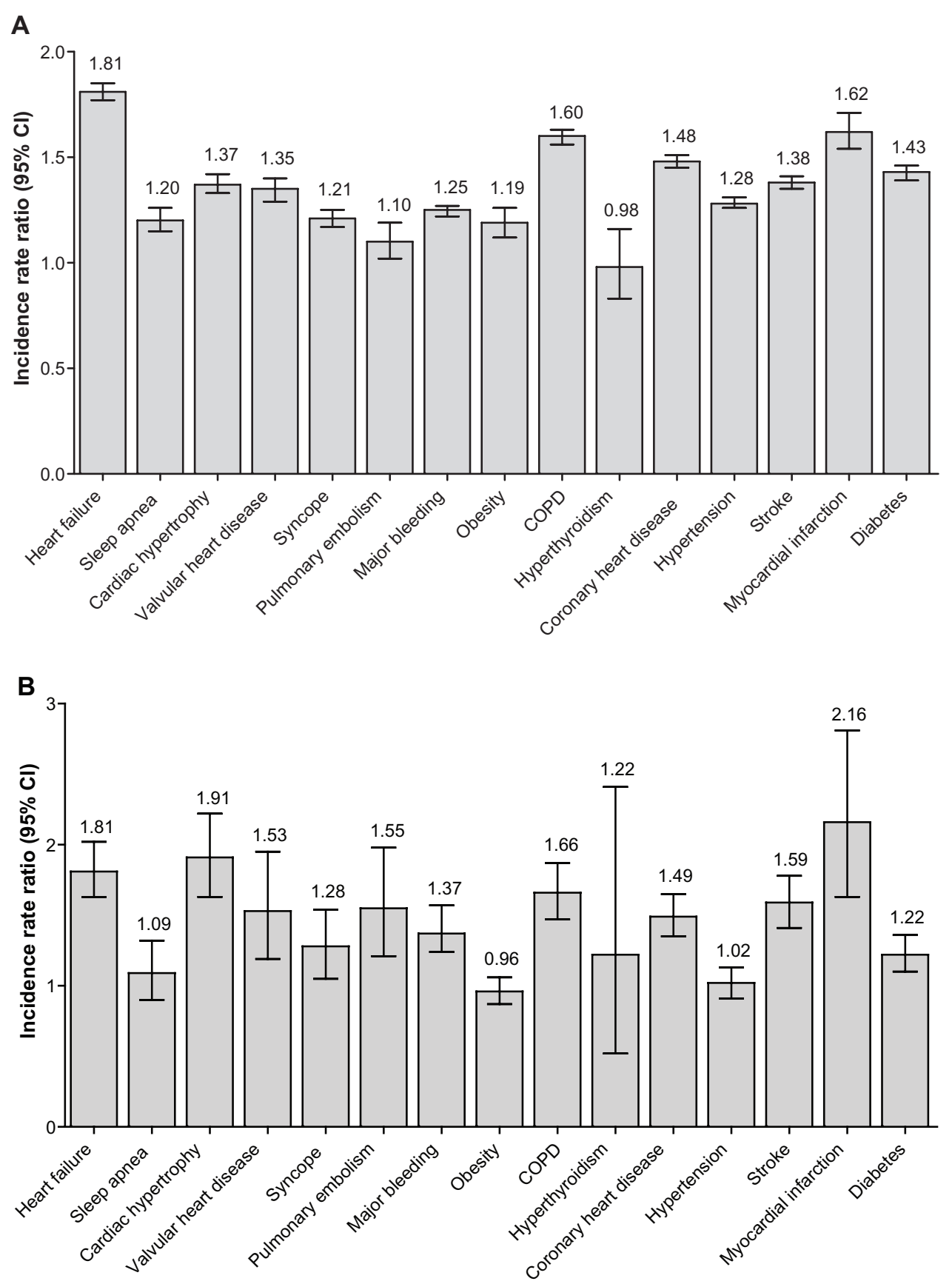

Figure 4 Comparison of incidence rates of cardiovascular hospitalization during the postindex period among AF patients with and without baseline comorbidity. Notes: (A) MS-Claims data set. (B) MG-EMR data set. Incidence rate ratio is the ratio between incidence rate in AF patients with baseline comorbidity and incidence rate in $\mathrm{AF}$ patients without baseline comorbidity.

Abbreviations: AF, atrial fibrillation/flutter; Cl, confidence interval; COPD, chronic obstructive pulmonary disease; MG-EMR, Geisinger Health System's MedMining electronic medical record database; MS-Claims, Truven Health Analytics MarketScan ${ }^{\circledR}$ claims databases.

scores (data not shown). IRRs (AF:non-AF patients) showed the opposite trend, reaching their highest value at $\mathrm{CHADS}_{2}$ and $\mathrm{CHA}_{2} \mathrm{DS}_{2}-\mathrm{VASc}$ scores of 0 (IRR $=4.86$ and 6.46 , respectively) and their lowest value at $\mathrm{CHADS}_{2}$ and $\mathrm{CHA}_{2} \mathrm{DS}_{2}-\mathrm{VASc}$ scores of 6 and 9 (IRR $=1.21$ and 1.12, respectively; Figure 6), indicating that the excess risk of cardiovascular hospitalization attributable to AF decreases as the comorbidity burden increases.

\section{Postindex risk of all-cause mortality in the EMR database}

The incidence rate of all-cause mortality during the follow-up period was $46 \%$ higher among AF patients than non-AF patients (Table 4). All-cause mortality risk among AF patients was increased $>2$-fold in the presence of MI, COPD, cardiac hypertrophy, heart failure, and stroke (Figure 7). 
Table 5 Incidence rates of new morbidity events and conditions during the follow-up period among AF and non-AF cohorts by database

\begin{tabular}{|c|c|c|c|c|c|c|}
\hline \multirow{3}{*}{$\begin{array}{l}\text { New morbidity } \\
\text { events or conditions }\end{array}$} & \multicolumn{3}{|l|}{ MS-Claims } & \multicolumn{3}{|l|}{ MG-EMR } \\
\hline & \multicolumn{2}{|c|}{$\begin{array}{l}\text { Incidence rate (n per } \\
\text { I,000 patient-years) }\end{array}$} & \multirow{2}{*}{$\begin{array}{l}\text { Incidence } \\
\text { rate ratio } \\
(95 \% \mathrm{Cl})\end{array}$} & \multicolumn{2}{|c|}{$\begin{array}{l}\text { Incidence rate (n per } \\
\text { I,000 patient-years) }\end{array}$} & \multirow{2}{*}{$\begin{array}{l}\text { Incidence } \\
\text { rate ratio } \\
(95 \% \mathrm{Cl})\end{array}$} \\
\hline & $\begin{array}{l}\text { AF cohort } \\
(n=105,262)\end{array}$ & $\begin{array}{l}\text { Non-AF cohort } \\
(n=105,262)\end{array}$ & & $\begin{array}{l}\text { AF cohort } \\
(n=3,9 \mid 9)\end{array}$ & $\begin{array}{l}\text { Non-AF cohort } \\
(n=3,872)\end{array}$ & \\
\hline Heart failure & 135.6 & 32.2 & $4.2(4.1-4.3)$ & 140.2 & 33.1 & $4.2(3.6-4.9)$ \\
\hline Cardiac hypertrophy & 66.8 & 18.0 & $3.7(3.6-3.9)$ & 77.9 & 22.9 & $3.4(2.8-4.1)$ \\
\hline Sleep apnea & 33.4 & 9.3 & $3.6(3.4-3.8)$ & 54.4 & 15.2 & $3.6(2.9-4.5)$ \\
\hline Valvular heart disease & 129.4 & 39.3 & $3.3(3.2-3.4)$ & 122.0 & 42.8 & $2.9(2.5-3.3)$ \\
\hline Pulmonary embolism & 10.7 & 3.6 & $3.0(2.7-3.3)$ & 14.0 & 6.1 & $2.3(1.6-3.4)$ \\
\hline Coronary heart disease & 170.2 & 58.9 & $2.9(2.8-3.0)$ & 137.3 & 76.4 & $1.8(1.6-2.0)$ \\
\hline Hyperthyroidism & 8.3 & 3.5 & $2.3(2.1-2.6)$ & 8.6 & 4.6 & $1.9(1.2-2.9)$ \\
\hline COPD & 79.9 & 37.6 & $2.1(2.1-2.2)$ & 72.4 & 38.1 & $1.9(1.6-2.2)$ \\
\hline Syncope & 55.4 & 27.1 & $2.0(2.0-2.1)$ & 49.5 & 19.8 & $2.5(2.1-3.1)$ \\
\hline Myocardial infarction & 17.7 & 8.9 & $2.0(1.9-2.1)$ & 15.4 & 10.7 & $1.4(1.1-2.0)$ \\
\hline Hypertension & 386.8 & 198.9 & $1.9(1.9-2.0)$ & 367.4 & 204.I & $1.8(1.6-2.0)$ \\
\hline Stroke & 113.3 & 58.6 & $1.9(1.9-2.0)$ & 104.2 & 63.4 & $1.6(1.4-1.9)$ \\
\hline Obesity & 14.3 & 7.5 & $1.9(1.8-2.1)$ & 56.6 & 25.9 & $2.2(1.8-2.6)$ \\
\hline Major bleeding & 313.4 & 166.9 & $1.9(1.9-1.9)$ & 315.9 & 140.1 & $2.3(2.1-2.5)$ \\
\hline Diabetes & 61.9 & 38.0 & I.6 (I.6-I.7) & 58.6 & 62.7 & $0.9(0.8-1.1)$ \\
\hline
\end{tabular}

Abbreviations: AF, atrial fibrillation/flutter; Cl, confidence interval; COPD, chronic obstructive pulmonary disease; MG-EMR, Geisinger Health System's MedMining electronic medical record database; MS-Claims, Truven Health Analytics MarketScan ${ }^{\circledast}$ claims databases.

\section{Discussion}

This descriptive study utilized a CDM to enable the analysis and comparison of two disparate data sources (administrative claims and EMR data). Markedly higher cardiovascular hospitalization and all-cause mortality rates were observed among patients with newly diagnosed AF compared with demographically similar patients without AF.

The retrospective, observational nature of the MS-Claims and MG-EMR analyses has implications for interpreting the study findings. Similar types of retrospective cohort studies have been previously described using claims data and matched non-AF controls; ${ }^{1,17}$ however, to our knowledge, this is the first analysis in which a CDM, particularly using EMR data, and standardized methods have been used to define AF/non-AF cohorts to examine both cardiovascular hospitalization and mortality outcomes.

The MS-Claims analysis was based on administrative claims data. Diagnostic and pharmacy prescription claims from billing records alone may not be reliable substitutes for clinically confirmed diagnoses, as there can be potential for coding inaccuracy. In contrast, the MG-EMR analysis was based on automated EMR records derived from medical practice. Advantages of the MG-EMR database include its comprehensive coverage of the elderly, the accuracy of its
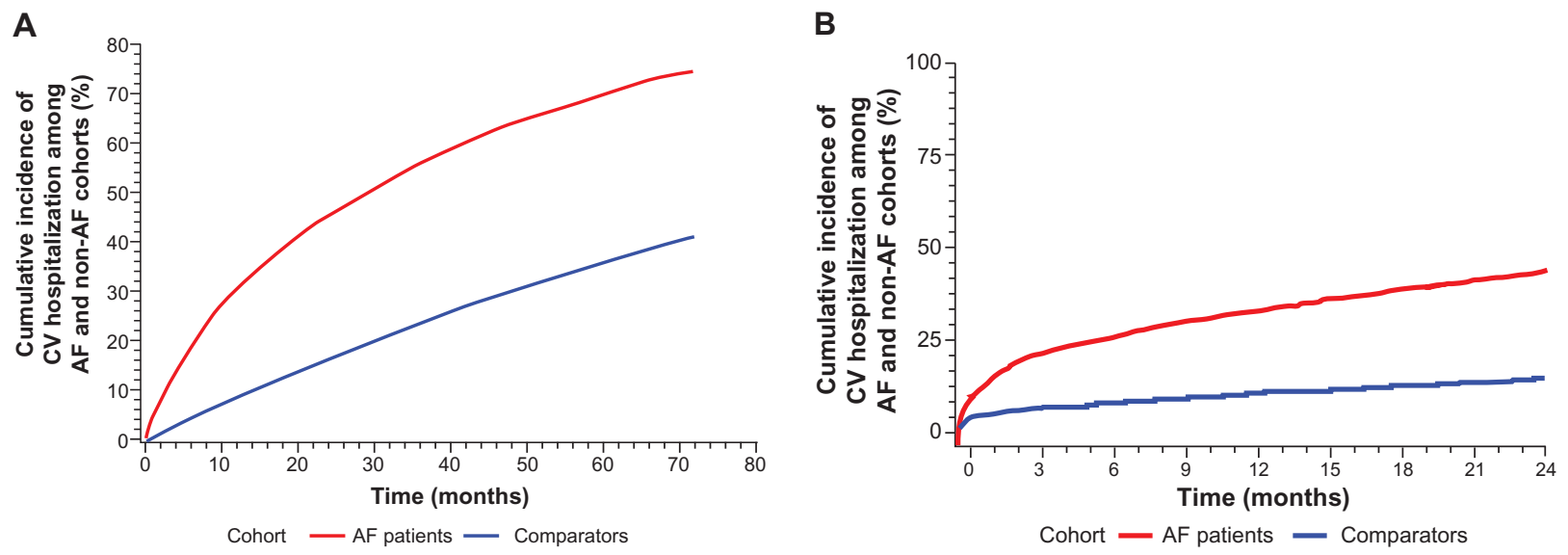

Figure 5 Kaplan-Meier estimates showing cumulative incidence of cardiovascular hospitalization over time among AF and non-AF patients. Notes: (A) MS-Claims data set. (B) MG-EMR data set.

Abbreviations: AF, atrial fibrillation/flutter; MG-EMR, Geisinger Health System's MedMining electronic medical record database; CV, cardiovascular; MS-Claims, Truven Health Analytics MarketScan ${ }^{\circledR}$ claims databases. 


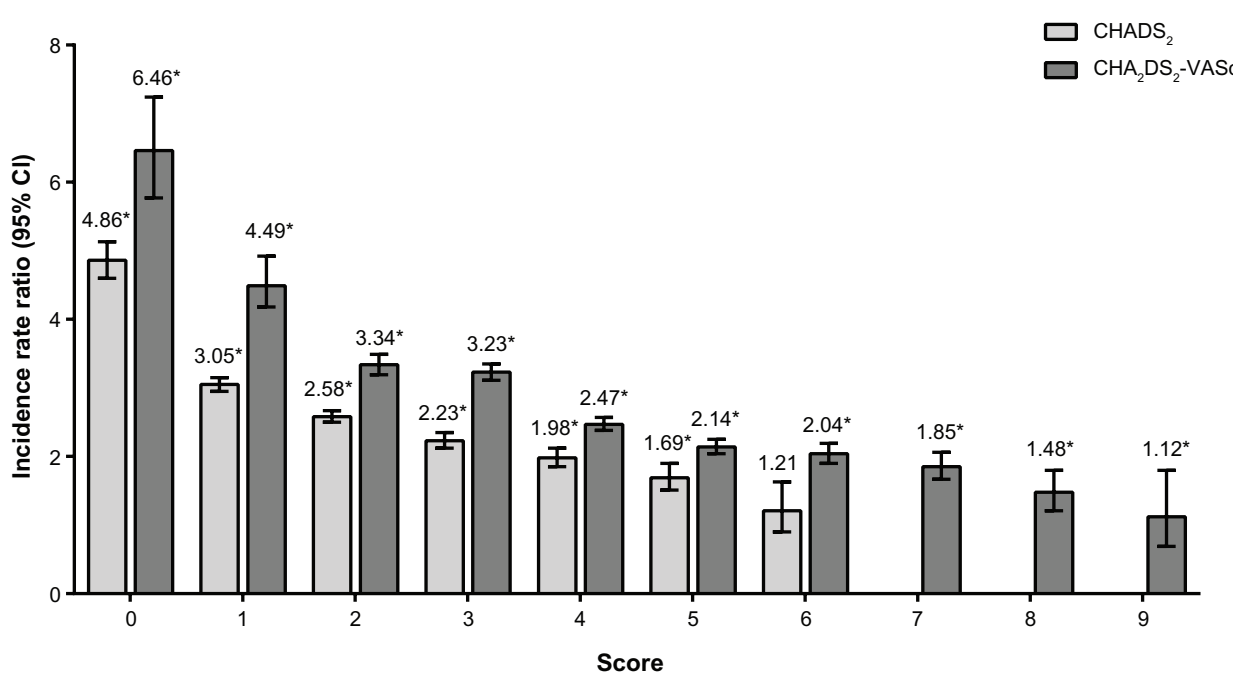

Figure 6 Comparison of incidence rates of cardiovascular hospitalization during the postindex period among AF and non-AF patients stratified by baseline $\mathrm{CHADS}_{2}$ and $\mathrm{CHA}_{2} \mathrm{DS}_{2}$-VASc scores (MS-Claims data set).

Note: Incidence rate ratio indicates the ratio between incidence rate in AF patients with baseline comorbidity and incidence rate in AF patients without baseline comorbidity. $* P<0.000$ I, $A F$ patients versus non-AF patients.

Abbreviations: AF, atrial fibrillation/flutter; $\mathrm{CHADS}_{2}$, Congestive heart failure, Hypertension, Age $\geq 75$ years, Diabetes mellitus, and prior Stroke/TIA; CHA $\mathrm{DS}_{2}-\mathrm{VASc}$, Congestive heart failure, Hypertension, Age $\geq 75$ years, Diabetes mellitus, prior Stroke/TIA, Vascular disease, Age 65-74 years, and Sex category; Cl, confidence interval; MS-Claims, Truven Health Analytics MarketScan ${ }^{\circledR}$ claims databases; TIA, transient ischemic attack.

coded records (MG-EMR data contain hospital discharge diagnoses that reflect the underlying medical reason for an inpatient stay), and its provision of vital status information that is unavailable from claims databases. Since hospitalization and mortality rates increase sharply with age, the MG-EMR analysis is expected to provide a more accurate estimate of these outcomes in AF patients. One drawback of the MG-EMR database is its narrow geographic representation (Central Pennsylvania, USA), meaning that the findings of the MG-EMR analysis, unlike the MS-Claims analysis, cannot be extrapolated nationally. Since Geisinger covers a large rural area where there are few alternative sources of health care,

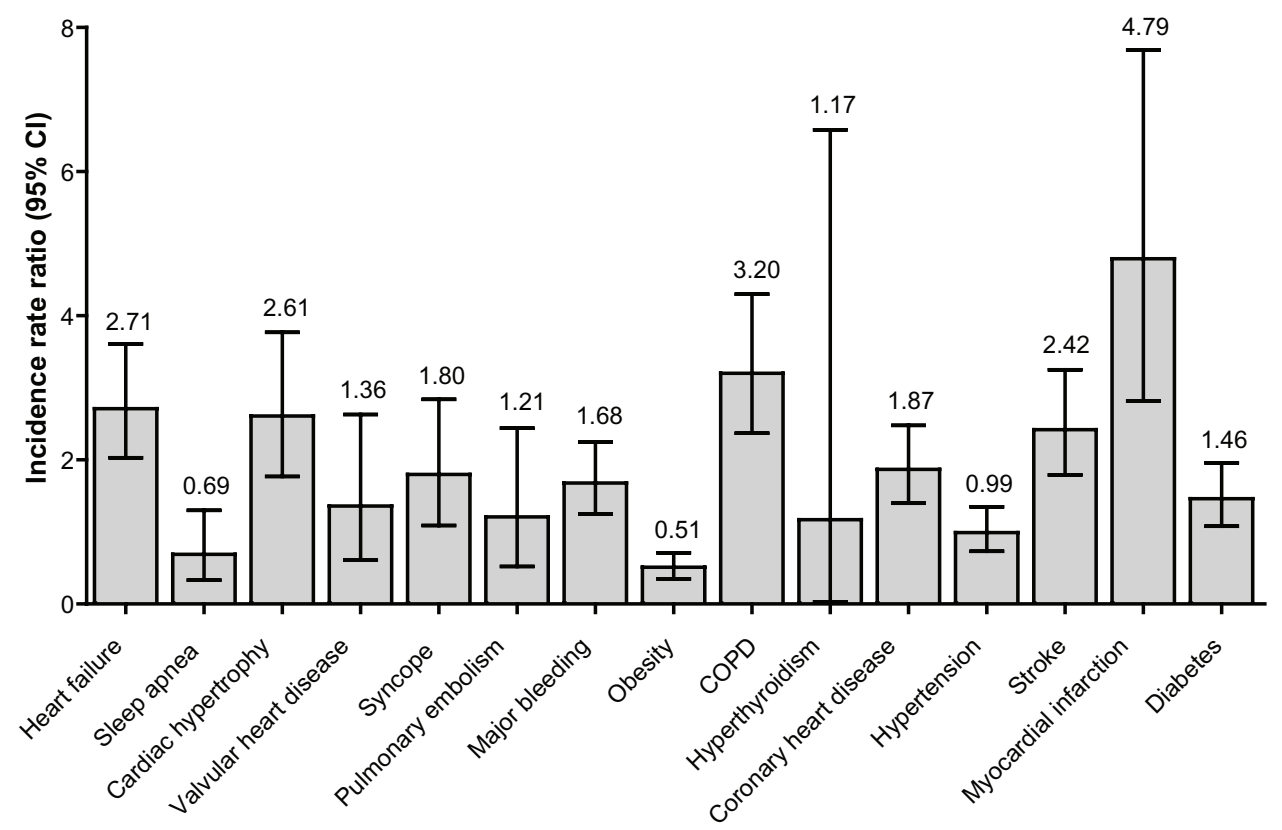

Figure 7 Comparison of incidence rates of all-cause mortality during the postindex period among AF patients with and without baseline comorbidity (MG-EMR data set). Note: Incidence rate ratio indicates the ratio between incidence rate in AF patients with baseline comorbidity and incidence rate in AF patients without baseline comorbidity. Abbreviations: AF, atrial fibrillation/flutter; Cl, confidence interval; COPD, chronic obstructive pulmonary disease; MG-EMR, Geisinger Health System's MedMining electronic medical record database. 
it is likely that most patients in the database receive their entire health care at Geisinger. However, as with some other EMR systems, it is possible for patients to receive health care outside the Geisinger system, which can potentially result in less than complete ascertainment of the number of health care encounters and hospitalizations. Nevertheless, the hospitalization rates in this study are comparable to other studies.

Despite the differences in geographic coverage, discharge and diagnostic codes, and comorbidity rates between the MS-Claims and MG-EMR databases, the IRRs for cardiovascular hospitalization in the two data sets were similar. Standardized analyses in both data sets showed that in the 12-month period prior to their index diagnosis, AF patients were in poorer health than their non-AF counterparts, shown by their higher prevalence of cardiovascular and metabolic comorbidities and AF-related symptoms. Analysis of the MS-Claims and MG-EMR data sets showed that MI was the baseline comorbidity most strongly associated with AF. Consistent with this, AF patients were much more likely to be prescribed anticoagulants, $\beta$-blockers, and digoxin during this prediagnostic period than demographically similar non-AF patients. Recognized risk factors for the development of AF include sleep apnea, pulmonary embolism, COPD, hyperthyroidism, obesity, and diabetes, ${ }^{4,5,30,31}$ conditions more common in the elderly, in whom AF is most prevalent.

Certain baseline comorbidities among AF patients appear to be captured at higher rates in the MG-EMR database compared with the MS-Claims database, particularly obesity, sleep apnea, pulmonary embolism, hypertension, and diabetes. The EMR data had a large percentage $(42 \%)$ of missing BMI and laboratory values (labs), with an imbalance between $\mathrm{AF}(23 \% \mathrm{BMI} ; \geq 20 \%$ labs) and non-AF patients ( $61 \% \mathrm{BMI} ; \geq 75 \%$ labs). This pattern is likely due to poor health status of the AF population, who would have had more health care encounters and associated records in the database than non-AF patients. The EMR data provided all-cause mortality data, but it was not possible to ascertain the specific cause of death without death certificate review. We expect the sensitivity of the administration claims data to be generally lower than that of medical records data. It is possible that patients are diagnosed clinically (in medical records), but this is not recorded in claims because treatments are not initiated. The differences across the databases observed in certain baseline medications reflect the variations in capture. EMR data capture prescribed medications, although these prescriptions are not necessarily filled or taken $^{32}$ and, therefore, reflect physician-prescribing patterns more accurately than claims data, which capture prescription medications that are dispensed to the patient. Furthermore, certain drugs, particularly those that are administered in hospitals (eg, heparin) or over-the-counter medications (eg, aspirin), may be underreported in claims data.

With regard to age differences between data sets, there was a greater concentration of older patients in MS-Claims due to an increased enrichment of Medicare patients, compared with MG-EMR.

Consistent with previous reports that $\mathrm{AF}$ itself predisposes toward development of other forms of cardiovascular disease, notably stroke and heart failure, ${ }^{3-6}$ this study demonstrated a high absolute incidence of de novo cardiovascular morbidity following AF diagnosis. Patients with newly diagnosed AF were at greater risk of developing a spectrum of cardiovascular, cardiometabolic, and respiratory comorbidities than non-AF patients. The high burden of preexisting and newly recorded morbidity was reflected in the higher rates of cardiovascular hospitalization ( $>3$-fold increase) and all-cause mortality ( $\sim 50 \%$ increase) among AF patients compared with non-AF patients. Of the baseline (prediagnostic) comorbidities evaluated, heart failure, cardiac hypertrophy, MI, COPD, coronary heart disease, and stroke appeared to have most impact in increasing cardiovascular hospitalization and allcause mortality rates following AF diagnosis.

A recent US study showed that the 3-year incidence of cardiovascular hospitalization ranged between $36 \%$ and $50 \%{ }^{33}$ The risk of cardiovascular hospitalization was increased by preexisting heart failure, coronary artery disease, or diabetes, regardless of the individual rhythm-control agent used. ${ }^{33}$ The ATHENA study, which prospectively evaluated the effect of dronedarone on cardiovascular hospitalization and all-cause mortality in paroxysmal/persistent AF, reported an incidence of first cardiovascular hospitalization of $~ 30 \%-40 \%$ over a follow-up period of 21 months. ${ }^{34}$

Few studies have evaluated how the comorbidity associated with new-onset AF relates to clinical prognosis in the real-world setting. The AnTicoagulation and Risk factors in Atrial Fibrillation, a cross-sectional database study of AF patients $(n=19,974)$, reported that, in the 5-year period prior to index diagnosis, the most prevalent comorbidities were hypertension (49\%), coronary heart disease (35\%), heart failure (29\%), and diabetes (17\%). ${ }^{35}$ International AF cohort studies in the UK and France have also reported a high prevalence of cardiovascular comorbidity and burden of symptoms among AF patients. ${ }^{36-38}$ The Registry on Cardiac Rhythm Disorders Assessing the Control of Atrial Fibrillation, a 1-year longitudinal study of patients with newly diagnosed paroxysmal/persistent $\mathrm{AF}(\mathrm{n}=5,604)$, reported that the 
presence of comorbid cardiovascular disease, renal disease, or $\mathrm{CHADS}_{2}$ score $\geq 2$ in the previous year was associated with cardiovascular hospitalization and mortality. ${ }^{39}$ Baseline symptoms predicted an adverse outcome independent of associated cardiovascular morbidity. ${ }^{39} \mathrm{~A}$ retrospective cohort study based on administrative claims (MarketScan ${ }^{\circledR}$ ) data identified $\mathrm{CHADS}_{2}$ score and $\mathrm{CHA}_{2} \mathrm{DS}_{2}$-VASc score as being the top two predictors of first cardiovascular hospitalization after AF diagnosis. ${ }^{40}$ Hospitalization risk increased two- to threefold in patients with a $\mathrm{CHADS}_{2}$ score of 6 , and threefold in patients with a $\mathrm{CHA}_{2} \mathrm{DS}_{2}$-VASc score of 9 compared with patients with scores of 0 , suggesting that these indices may be useful in identifying at-risk AF patients.

This study has some notable limitations. For both analyses, information on the frequency, duration, and symptoms associated with AF were unavailable, and the extent to which cardiovascular hospitalization and mortality were affected by the clinical setting and treatment is unclear. Despite the balance in baseline demographics between the AF and non-AF cohorts, there is a likelihood of bias due to imbalance in nonidentified covariates. Since the study objective was to utilize a CDM to describe patterns of clinical risk factors and cardiovascular hospitalization risk across disparate data sets, regression analysis with multivariate adjustment for associated comorbidities and health status was not performed. Hence, causal relationships between the individual baseline comorbidities and either cardiovascular hospitalization or mortality are neither assumed nor inferred. It was not possible to distinguish between paroxysmal, persistent, and permanent $\mathrm{AF}$ in this study as this information was not available in the databases used, in common with previous retrospective cohort studies. ${ }^{41-43}$ This study did, however, identify patients with chronic AF by excluding patients with transient AF.

In conclusion, by conducting similar standardized analyses in two data sources, this study indicates consistency with other primary data sources that AF is associated with acute morbidity and increased risk of cardiovascular hospitalization and all-cause mortality. AF management and treatment remains an unmet medical need, evidenced by the high prevalence of comorbidity at the time of AF diagnosis and by the association between this comorbidity and the risk of subsequent cardiovascular hospitalization, and all-cause mortality following diagnosis of AF.

\section{Acknowledgments}

The study was funded by Sanofi. Editorial support for manuscript development was provided by Andrew Fitton, $\mathrm{PhD}$, and Sharmila Blows, PhD, of Excel Scientific Solutions.

\section{Disclosure}

Mary P Panaccio, Raymond Miao, and Andrew Koren are employees of Sanofi, USA. Gordon Cummins is an employee of Quintiles, which has a research consultancy agreement with Sanofi. At the time of this study, Charles Wentworth, Stephan Lanes, Shannon L Reynolds and Matthew W Reynolds were employees of United BioSource Corporation, which had a research consultancy agreement with Sanofi.

Part of the data from the manuscript was previously presented in abstract and poster form at the International Society for Pharmacoeconomics and Outcomes Research and the American Public Health Association 2012 annual meetings.

The authors have no other conflicts of interest to disclose.

\section{References}

1. Naccarelli GV, Varker H, Lin J, Schulman KL. Increasing prevalence of atrial fibrillation and flutter in the United States. Am J Cardiol. 2009;104(11):1534-1539.

2. Halligan SC, Gersh BJ, Brown RD Jr, et al. The natural history of lone atrial flutter. Ann Intern Med. 2004;140(4):265-268.

3. Wolf PA, Abbott RD, Kannel WB. Atrial fibrillation as an independent risk factor for stroke: the Framingham Study. Stroke. 1991;22(8): 983-988.

4. Sanoski CA. Prevalence, pathogenesis, and impact of atrial fibrillation. Am J Health Syst Pharm. 2010;67(9 Suppl 5):S11-S16.

5. Lakshminarayan K, Anderson DC, Herzog CA, Qureshi AI. Clinical epidemiology of atrial fibrillation and related cerebrovascular events in the United States. Neurologist. 2008;14(3):143-150.

6. Stewart S, Hart CL, Hole DJ, McMurray JJ. A population-based study of the long-term risks associated with atrial fibrillation: 20-year follow-up of the Renfrew/Paisley study. Am J Med. 2002;113(5):359-364.

7. Miyasaka Y, Barnes ME, Bailey KR, et al. Mortality trends in patients diagnosed with first atrial fibrillation: a 21-year community-based study. J Am Coll Cardiol. 2007;49(9):986-992.

8. Crandall MA, Horne BD, Day JD, et al. Atrial fibrillation significantly increases total mortality and stroke risk beyond that conveyed by the CHADS2 risk factors. Pacing Clin Electrophysiol. 2009;32(8): 981-986.

9. Go AS, Mozaffarian D, Roger VL, et al. Heart disease and stroke statistics - 2013 update: a report from the American Heart Association. Circulation. 2013;127(1):e6-e245.

10. Lip GY, Tean KN, Dunn FG. Treatment of atrial fibrillation in a district general hospital. Br Heart J. 1994;71(1):92-95.

11. Zarifis J, Beevers G, Lip GY. Acute admissions with atrial fibrillation in a British multiracial hospital population. Br J Clin Pract. 1997;51(2): 91-96.

12. Miyasaka Y, Barnes ME, Gersh BJ, et al. Secular trends in incidence of atrial fibrillation in Olmsted County, Minnesota, 1980 to 2000, and implications on the projections for future prevalence. Circulation. 2006;114(2):119-125.

13. Khairallah F, Ezzedine R, Ganz LI, London B, Saba S. Epidemiology and determinants of outcome of admissions for atrial fibrillation in the United States from 1996 to 2001. Am J Cardiol. 2004;94(4): $500-504$.

14. Miyasaka Y, Barnes ME, Gersh BJ, et al. Changing trends of hospital utilization in patients after their first episode of atrial fibrillation. $A m J$ Cardiol. 2008;102(5):568-572.

15. Friberg J, Buch P, Scharling H, Gadsbphioll N, Jensen GB. Rising rates of hospital admissions for atrial fibrillation. Epidemiology. 2003;14(6): 666-672. 
16. Wattigney WA, Mensah GA, Croft JB. Increasing trends in hospitalization for atrial fibrillation in the United States, 1985 through 1999: implications for primary prevention. Circulation. 2003;108(6): 711-716.

17. Kim MH, Johnston SS, Chu B-C, Dalal MR, Schulman KL. Estimation of total incremental health care costs in patients with atrial fibrillation in the United States. Circ Cardiovasc Qual Outcomes. 2011;4(3): 313-320.

18. Coyne KS, Paramore C, Grandy S, Mercader M, Reynolds M, Zimetbaum P. Assessing the direct costs of treating nonvalvular atrial fibrillation in the United States. Value Health. 2006;9(5):348-356.

19. Kim MH, Klingman D, Lin J, Pathak P, Battleman DS. Cost of hospital admission for antiarrhythmic drug initiation in atrial fibrillation. Ann Pharmacother. 2009;43(5):840-848.

20. Ye X, Gross CR, Schommer J, Cline R, Xuan J, St Peter WL. Initiation of statins after hospitalization for coronary heart disease. J Manag Care Pharm. 2007;13(5):385-396.

21. Amin A, Stemkowski S, Lin J, Yang G. Thromboprophylaxis rates in US medical centers: success or failure? J Thromb Haemost. 2007;5(8): 1610-1616.

22. Chapman RH, Petrilla AA, Berman L, Benner JS, Tang SS. Are highrisk hypertensive patients being prescribed concomitant statin therapy? A retrospective cohort study. Am J Cardiovasc Drugs. 2009;9(5): 299-308.

23. Herout PM, Harshaw Q, Phatak H, et al. Impact of worsening renal function during hospital admission on resource utilization in patients with heart failure. Am J Cardiol. 2010;106(8):1139-1145.

24. Asche C, Nelson R, McAdam-Marx C, Jhaveri M, Ye X. Predictors of oral bisphosphonate prescriptions in post-menopausal women with osteoporosis in a real-world setting in the USA. Osteoporos Int. 2010;21(8):1427-1436.

25. Nordstrom BL, Kachroo S, Fraeman KH, et al. Warfarin prophylaxis in patients after total knee or hip arthroplasty - international normalized ratio patterns and venous thromboembolism. Curr Med Res Opin. 2011;27(10):1973-1985.

26. Reisinger SJ, Ryan PB, O'Hara DJ, et al. Development and evaluation of a common data model enabling active drug safety surveillance using disparate healthcare databases. J Am Med Inform Assoc. 2010;17(6): 652-662.

27. Charlson ME, Pompei P, Ales KL, MacKenzie CR. A new method of classifying prognostic comorbidity in longitudinal studies: development and validation. J Chronic Dis. 1987;40(5):373-383.

28. Gage BF, Waterman AD, Shannon W, Boechler M, Rich MW, Radford MJ. Validation of clinical classification schemes for predicting stroke: results from the National Registry of Atrial Fibrillation. JAMA. 2001;285(22):2864-2870

29. Lip GY, Nieuwlaat R, Pisters R, Lane DA, Crijns HJ. Refining clinical risk stratification for predicting stroke and thromboembolism in atrial fibrillation using a novel risk factor-based approach: the euro heart survey on atrial fibrillation. Chest. 2010;137(2):263-272.
30. Callans DJ. In the clinic. Atrial fibrillation. Ann Intern Med. 2008;149(9):ITC5-1-15

31. Benjamin EJ, Chen PS, Bild DE, et al. Prevention of atrial fibrillation: report from a national heart, lung, and blood institute workshop. Circ Cardiovasc Qual Outcomes. 2009;119(4):606-618.

32. Moore M, Yuen HM, Dunn N, Mullee MA, Maskell J, Kendrick T. Explaining the rise in antidepressant prescribing: a descriptive study using the general practice research database. BMJ. 2009;339:b3999.

33. Saksena S, Slee A, Waldo AL, et al. Cardiovascular outcomes in the AFFIRM Trial (atrial fibrillation follow-up investigation of rhythm management). An assessment of individual antiarrhythmic drug therapies compared with rate control with propensity score-matched analyses. J Am Coll Cardiol. 2011;58(19):1975-1985.

34. Hohnloser SH, Crijns HJ, van Eickels M, et al; ATHENA Investigators. Effect of dronedarone on cardiovascular events in atrial fibrillation. N Engl J Med. 2009;360(7):668-678.

35. Go AS, Hylek EM, Borowsky LH, Phillips KA, Selby JV, Singer DE. Warfarin use among ambulatory patients with nonvalvular atrial fibrillation: the Anticoagulation and Risk Factors in Atrial Fibrillation (ATRIA) study. Ann Intern Med. 1999;131(12):927-934.

36. Carroll K, Majeed A. Comorbidity associated with atrial fibrillation: a general practice-based study. Br J Gen Pract. 2001;51(472): 884-886, 889-891.

37. Cohen A, Dallongeville J, Durand-Zaleski I, Bouee S, Le Heuzey JY. Characteristics and management of outpatients with history of or current atrial fibrillation: the observational French EPHA study. Arch Cardiovasc Dis. 2010;103(6-7):376-387.

38. Lévy S, Maarek M, Coumel P, et al. Characterization of different subsets of atrial fibrillation in general practice in France: the ALFA study. The College of French Cardiologists. Circulation. 1999;99(23):3028-3035.

39. Camm AJ, Breithardt G, Crijns H, et al. Real-life observations of clinical outcomes with rhythm- and rate-control therapies for atrial fibrillation RECORDAF (Registry on Cardiac Rhythm Disorders Assessing the Control of Atrial Fibrillation). J Am Coll Cardiol. 2011;58(5): 493-501.

40. Naccarelli GV, Panaccio MP, Cummins G, Tu N. CHADS2 and CHA2DS2-VASc risk factors to predict first cardiovascular hospitalization among atrial fibrillation/atrial flutter patients. Am J Cardiol. 2012;109(10):1526-1533.

41. Naccarelli GV, Johnston SS, Lin J, Patel PP, Schulman KL. Cost burden of cardiovascular hospitalization and mortality in ATHENA-like patients with atrial fibrillation/atrial flutter in the United States. Clin Cardiol. 2010;33(5):270-279.

42. Amin AN, Jhaveri M, Lin J. Incremental cost burden to US healthcare payers of atrial fibrillation/atrial flutter patients with additional risk factors. Adv Ther. 2011;28(10):907-926.

43. Kim MH, Lin J, Jhaveri M, Koren A. Impact of dronedarone treatment on healthcare resource utilization in patients with atrial fibrillation/ flutter. Adv Ther. 2014;31(3):318-332.
Clinical Epidemiology

\section{Publish your work in this journal}

Clinical Epidemiology is an international, peer-reviewed, open access, online journal focusing on disease and drug epidemiology, identification of risk factors and screening procedures to develop optimal preventative initiatives and programs. Specific topics include: diagnosis, prognosis, treatment, screening, prevention, risk factor modification,

Submit your manuscript here: http://www.dovepress.com/clinical-epidemiology-journal

\section{Dovepress}

systematic reviews, risk \& safety of medical interventions, epidemiology \& biostatistical methods, and evaluation of guidelines, translational medicine, health policies \& economic evaluations. The manuscript management system is completely online and includes a very quick and fair peer-review system, which is all easy to use. 